\title{
Baicalin Attenuates Alcoholic Liver Injury through Modulation of Hepatic Oxidative Stress, Inflammation and Sonic Hedgehog Pathway in Rats
}

\author{
Huifen Wang Yanli Zhang Ruxue Bai Miao Wang Shiyu Du \\ Department of Gastroenterology, China-Japan Friendship Hospital, Beijng, People's Republic of China
}

\section{Key Words}

Baicalin • Alcoholic liver injury • Oxidative stress • Inflammation • Sonic hedgehog

\begin{abstract}
Background/Aims: Lipid accumulation, inflammatory responses and oxidative stress have been implicated in the pathology of alcoholic liver disease (ALD). Targeting inhibition of these features may provide a promising therapeutic strategy for ALD. Baicalin, a flavonoid isolated from Scutellaria baicalensis Georgi, has been shown to exert a hepatoprotective effect. However, its effects on ALD remain obscure. This study was aimed to investigate the effects of baicalin on alcohol-induced liver injury and its related mechanisms. Methods: For in vivo experiments, rats were supplied intragastrical administration of alcohol continuously for 4 or 8 weeks, and then received baicalin treatment in the latter 4 weeks in the presence / absence of alcohol intake. Liver histology and function, inflammatory cytokines, oxidative mediators, and the components of the Sonic hedgehog pathway were evaluated. For in vitro experiments, alcohol-stimulated human normal liver cells LO2 were used. Results: Baicalin treatment significantly alleviated alcoholic liver injury, improved liver function impaired by alcohol, and inhibited hepatocytes apoptosis. In addition, baicalin decreased the expression levels of proinflammatory cytokines (TNF- $\alpha$, IL-1 $\beta$, IL- 6 ) and malonyldialdehyde (MDA), and increased the activities of antioxidant enzymes SOD and GSH-Px. Furthermore, baicalin modulated the activation of Sonic hedgehog (Shh) pathway. Administration of baicalin upregulated the expression of sonic hedgehog (Shh), patched (Ptc), Smoothened (Smo), and Glioblastoma-1(Gli-1). Blockade of the Shh pathway in cyclopamine abolished the effects of baicalin in vitro. Conclusion: Both in vivo and in vitro experimental results indicate that baicalin exerts hepatoprotective roles in alcohol-induced liver injury through inhibiting oxidative stress, inflammatory response, and the regulation of the Shh pathway.
\end{abstract}

H. Wang and Y. Zhang contributed equally to this study.

Dr. Shiyu Du

KARGER
Department of Gastroenterology, China-Japan Friendship Hospital, 2 Yinghua Dongjie, Beijng 100029, (People's Republic of China)

Fax/Tel. +86-10-84205288, E-Mail shiyd_01@sina.com 


\section{Cellular Physiology Cell Physiol Biochem 2016;39:1129-1140 \begin{tabular}{ll|l} 
DOI: 10.1159/000447820 & $\begin{array}{l}\text { O 2016 The Author(s). Published by S. Karger AG, Basel } \\
\text { www.karger.com/cpb }\end{array}$ \\
\hline
\end{tabular} \\ Wang et al.: Baicalin Attenuates Alcoholic Liver Injury in Rats}

\section{Introduction}

Alcoholic liver disease (ALD) is one of the main causes of liver diseases impairing the health of citizens and is associated with a broad spectrum of liver disorders including steatosis, steatohepatitis, cirrhosis, and hepatocarcinoma [1,2]. Data from the National Institute on Alcohol Abuse and Alcoholism (NIAAA) show that there were approximately 75,766 of alcohol-related deaths during 2001 in the United States [3]. Thus, it is urgently to develop novel effective therapies to prevent and treat alcohol-related diseases. Despite the pathological mechanisms underlying ALD remain largely unknown, oxidative stress and inflammation have been considered as essential intracellular events contributing to ALD. Hence, targeting these major features may provide a promising therapeutic strategy for ALD.

Baicalin, a flavonoid isolated from the Scutellaria baicalensis Georgi, is known to possess multiple biological effects such as anti-oxidant [4], anti-inflammatory [5], and immunoregulatory properties [6]. Recent studies have shown that baicalin can protect liver from various detrimental factors induced damages, including carbon tetrachlorideinduced acute liver injury [7], iron or cadmium overload-induced hepatic cytotoxicity [8], and ischemia/reperfusion (I/R)-induced hepatocellular injury [9], suggesting a potent hepatoprotective agent of baicalin. Recently, Kim et al. [10] further showed that baicalin could attenuate I/R-induced inflammatory responses in alcoholic fatty liver condition, indicating that baicalin may exert therapeutic effects in alcoholic liver injury. Thereby, this study was conducted to investigate the potent protective effects of baicalin on ALD, as well as discussed the underlying mechanisms of its therapeutic action.

\section{Materials and Methods}

\section{Animals and treatment}

Male Wistar rats (weighing 180-220g) were housed in plastic cages with free access to food and water. Animals were kept at a temperature $22 \pm 2^{\circ} \mathrm{C}$ and a relative humidity of $50 \%$ environments. Animal experimental procedures were approved by the Experimental Animal Ethics Committee of China-Japan Friendship Institute and in accordance with the Guiding Principles for the Care and Use of Laboratory Animals. Rats were randomly assigned into five groups ( $\mathrm{n}=6$ in each group): Control group (no alcohol intake, no baicalin treatment), ALD model I group ( 8 weeks of alcohol intake, no baicalin treatment), ALD model II group (4 weeks of alcohol intake, no baicalin treatment), Baicalin treatment I group (8 weeks of alcohol intake, 4 weeks of baicalin treatment), Baicalin treatment II group (4 weeks of alcohol intake, 4 weeks of baicalin treatment). Except the control group, the remaining four groups were provided with alcohol by gavage. The dose of alcohol was $65 \%$ (vol $/ \mathrm{vol}$ ), $5 \mathrm{ml} / \mathrm{kg} / \mathrm{day}$ in the first three days, and then $65 \%$ (vol $/ \mathrm{vol}$ ), $10 \mathrm{ml} / \mathrm{kg} /$ day in the following days. After 4 weeks, the ALD I group was continuously supplied with alcohol for another 4 weeks; while the ALD II group stopped alcohol consumption. The baicalin I and baicalin II groups were intragastrically received baicalin at a concentration of $120 \mathrm{mg} / \mathrm{kg} /$ day (baicalin purity $\geq 98 \%$, Meilun Biotech, Dalian, China) for four weeks. The dosage of baicalin was based on the previous reports [10, $11]$ and our preliminary experiments. Animal body conditions were monitored through the experimental process. Rats were euthanized at 8 weeks, and the liver tissues and blood samples were collected for the following experiments.

\section{Biochemical assays}

Several biochemical indicators including alanine aminotransferase (ALT), aspartate aminotransferase (AST), and triglyceride (TG) are used in the diagnosis of liver diseases [12-14]. The activities of ALT and AST, and the content of TG were detected using commercial kits (Nanjing Jiancheng Bioengineering Research Institute) according to the instructions.

Liver histology

Liver tissues were fixed in $10 \%$ formalin and embedded in paraffin. Then the paraffin-embedded tissues were cut into $5 \mu \mathrm{m}$ thicknesses of sections. After being stained with hematoxylin and eosin (H\&E), 


\section{Cellular Physiology Cell Physiol Biochem 2016;39:1129-1140 \begin{tabular}{l|l|l}
\cline { 2 - 3 } DOI: 10.1159/000447820 & (c) 2016 The Author(s). Published by S. Karger AG, Basel
\end{tabular} and Biochemistry Published online: August 29, 2016 www.karger.com/cpb \\ Wang et al.: Baicalin Attenuates Alcoholic Liver Injury in Rats}

the liver tissue sections were examined microscopically to evaluate tissue damages using an OLYMPUSDP71 microscope.

\section{Measurement of TNF- $\alpha, I L-1 \beta$, and $I L-6$}

The concentration of TNF- $\alpha$, IL-1 $\beta$, and IL-6 in serum and liver tissue homogenate was measured using commercial enzyme-linked immunosorbent assay kits specific for rat according to the manufacturer's instructions (Boster, Wuhan, China).

\section{Measurement of MDA, SOD, and GSH content}

Liver tissue samples were homogenized. The supernatants of homogenate were used to determinate the levels of malonyldialdehyde (MDA), glutathione (GSH-Px) and superoxide dismutase (SOD) accordingly (Nanjing Jiancheng Bioengineering Institute, Nanjing, China).

\section{Western blot analysis}

Liver samples were homogenized and total proteins from the homogenate were extracted using NP-40 Lysis Buffer (Beyotime Institute of Biotechnology, Haimen, China), the protein concentration of each sample was determined using bicinchoninic acid method (Beyotime). Equal amounts of protein were separated by SDS-PAGE (10\% and 13\% gels) and the separated proteins were transferred onto polyvinylidene difluoride membranes (Millipore, Billerica, MA, USA). The membranes were incubated with primary antibodies against Shh, Smo, Ptc, Gli1, Bcl-2, Bax, and cleaved-caspase 3 at $4{ }^{\circ} \mathrm{C}$ overnight, respectively (anti-Shh and anti-Smo, Bioss, Beijing, China, 1:500 diluted; anti-Ptc, Sangon, Shanghai, China, 1:400 diluted; anti-Gli1, USCN life science, Wuhan, China, 1:200 diluted; anti-Bcl-2 and anti-Bax, Boster, Wuhan, China, 1:400 diluted; anticleaved-caspase 3, Abcam, Cambridge, MA, 1:1000 diluted). After washed three times, the membranes were again incubated with horseradish peroxidase (HRP)-labeled secondary antibody (1:5000 diluted, Beyotime) at $37^{\circ} \mathrm{C}$ for $45 \mathrm{~min}$. Protein blots on the membranes was visualized using electrochemiluminescence (ECL) reagents (7Sea Biotech, Shanghai, China), and the scanned images were analyzed with Gel-Pro-Analyzer software 4.0 (Media Cybernetics, Silver Spring, MD, USA).

\section{TUNEL staining}

Apoptotic cells in sections of liver tissues were detected using the terminal deoxynucleotidyl transferase (TdT)-mediated dUTP-biotin nick end labeling (TUNEL, Roche, Basel, Switzerland) according to the manufacturer's instructions.

\section{Immunohistochemistry}

The expressions of Shh, Smo, Ptch, and Gli1 in liver were examined by immunohistochemical staining. Briefly, slices of $5 \mu \mathrm{m}$ thicknesses were deparaffinized, and rehydrated, then the slices were performed for antigen retrieval using citrate buffer $\left(\mathrm{pH} \mathrm{6.0)}\right.$ ) at $100{ }^{\circ} \mathrm{C}$ for $10 \mathrm{~min}$ and placed in $3 \% \mathrm{H}_{2} \mathrm{O}_{2}$ for 10 min to exhaust endogenous peroxidase activity. After being blocked with goat serum for $30 \mathrm{~min}$, the slices were incubated with primary antibodies (anti-Shh, anti-Smo, and anti-Ptc were from Sangon biotech, Shanghai, China, 1:50 diluted; anti-Gli1, USCN life science, Wuhan, China, 1:200 diluted) at $4^{\circ} \mathrm{C}$ overnight. After washed three times in PBS, the slices were incubated were incubated with HRP-onjugated goat anti-rabbit secondary antibody (1:200 diluted) at $37^{\circ} \mathrm{C}$ for another $30 \mathrm{~min}$. At last, the slides were developed with diaminobenzidine and hematoxylin counterstaining and the immunohistochemistry changes were observed under a light microscope.

\section{Cell culture and treatments}

Human normal liver cell LO2 was obtained from Zhongqiaoxinzhou Biotech (Shanghai, China). The cells were cultured in Dulbecco's modified eagle medium (DMEM; Cibco, Grand Island, NY, USA) containing $10 \%$ fetal bovine serum (FBS; Hyclone, Logan, UT, USA). Cells were maintained in a humidified atmosphere of $5 \% \mathrm{CO} 2$ at $37^{\circ} \mathrm{C}$. The cells were exposed to $100 \mathrm{mM}$ alcohol in the absence / presence of baicalin $(25,50$ $\mu \mathrm{M})$ or cyclopamine $(20 \mu \mathrm{M})$ for $24 \mathrm{~h}$ and then harvested. The dosage of these drugs and alcohol were chosen by our preliminary experiments. 


\section{Cellular Physiology Cell Physiol Biochem 2016;39:1129-1140 \begin{tabular}{c|l|l} 
and Biochemistry & $\begin{array}{c}\text { DOI: 10.1159/000447820 } \\
\text { Published online: August 29, } 2016\end{array}$ & $\begin{array}{l}\text { O 2016 The Author(s). Published by S. Karger AG, Basel } \\
\text { www.karger.com/cpb }\end{array}$ \\
\cline { 2 - 3 }
\end{tabular} \\ Wang et al.: Baicalin Attenuates Alcoholic Liver Injury in Rats}

Hoechst 33258 fluorescence staining

LO2 cells were stimulated with alcohol in the absence / presence of baicalin $(25,50 \mu \mathrm{M})$ or cyclopamine $(20 \mu \mathrm{M})$ for $24 \mathrm{~h}$. The cells were fixed, then stained with Hoechst staining kits (Beyotime Institute of Biotechnology, Haimen, China) according to the manufacturer's instructions. Apoptotic cells were detected under a fluorescence microscope.

\section{Statistical analysis}

All values are expressed as the mean \pm standard deviation (SD), and data are processed in GraphPad Prism 5.0 software (GraphPad Software Inc, San Diego, CA, USA). Differences among groups were performed using analysis of variance (ANOVA), followed by the Bonferroni test for post hoc comparisons. A P value $<0.05$ was considered statistically significant.

\section{Results}

Baicalin alleviates alcohol-induced liver injury in rats

To evaluate the protective effects of baicalin on alcohol-induced liver injury in rats, we first examined the histological changes of liver tissues from five different groups. As shown in Fig. 1A, liver tissues in the control group exhibited normal lobular architecture that contained central veins and radiating hepatic cords. In contrast, live tissues of ALD groups

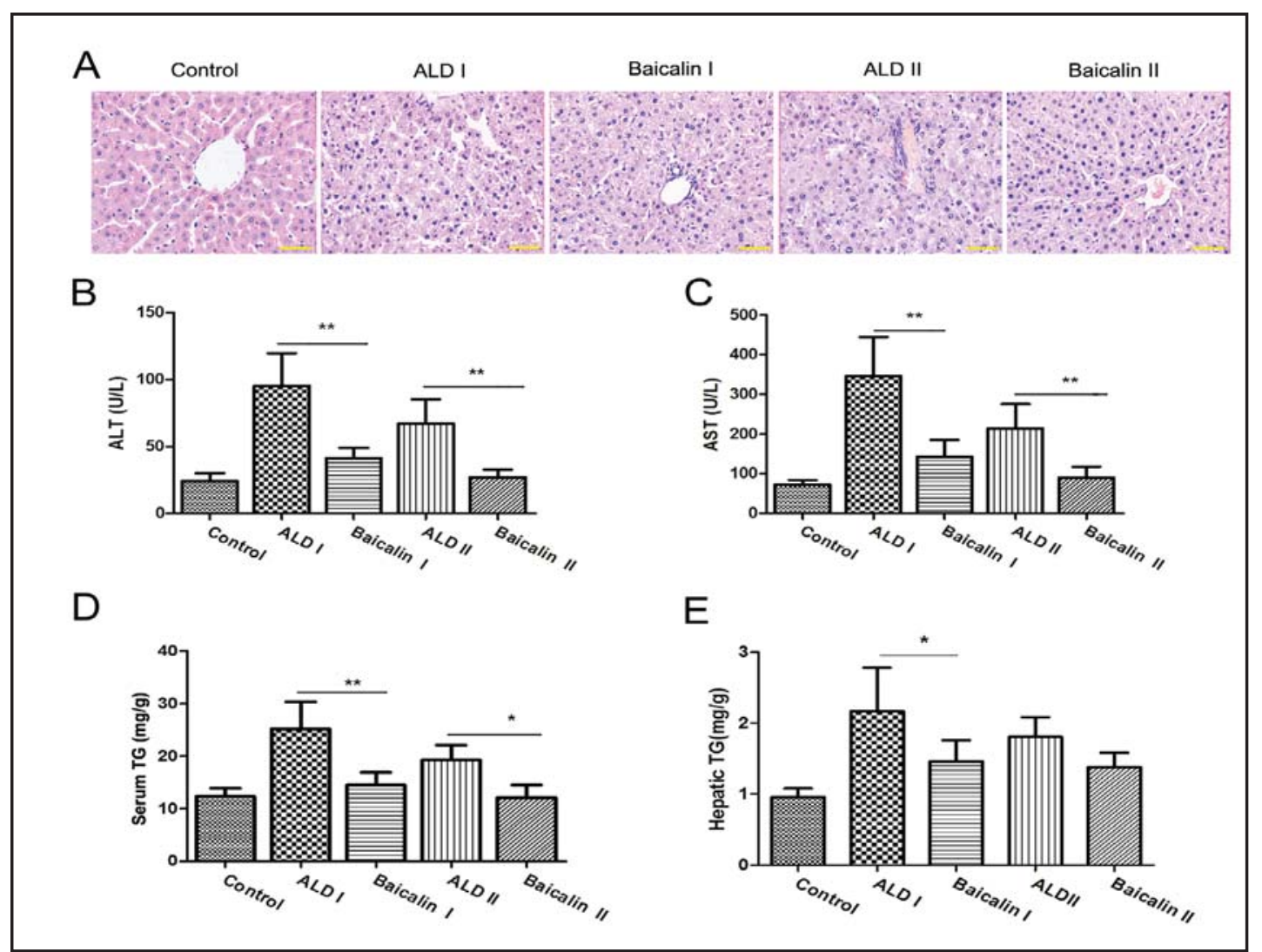

Fig. 1. Baicalin treatment alleviates alcohol-induced liver injury in rats. Rats were grouped: normal control (no alcohol, no treatment); ALD I model group (with alcohol 8 weeks, no treatment); Baicalin I treatment group (baicalin $120 \mathrm{mg} / \mathrm{kg}+$ alcohol 8 weeks); ALDII model group (with alcohol 4 weeks, no treatment); Baicalin II treatment group (baicalin $120 \mathrm{mg} / \mathrm{kg}+$ alcohol 4 weeks). (A) Liver histologic changes determined by hematoxylin and eosin staining. Magnification $\times 400$, scale bars $=50 \mu \mathrm{m}$. The serum levels of ALT (B), AST(C), TG (D), and hepatic TG (E) were determined. Data are presented as mean \pm SD. ${ }^{*} p<0.05$ and ${ }^{* *} p<0.01$ vs. ALD model group. 


\section{Cellular Physiology \\ Cell Physiol Biochem 2016;39:1129-1140

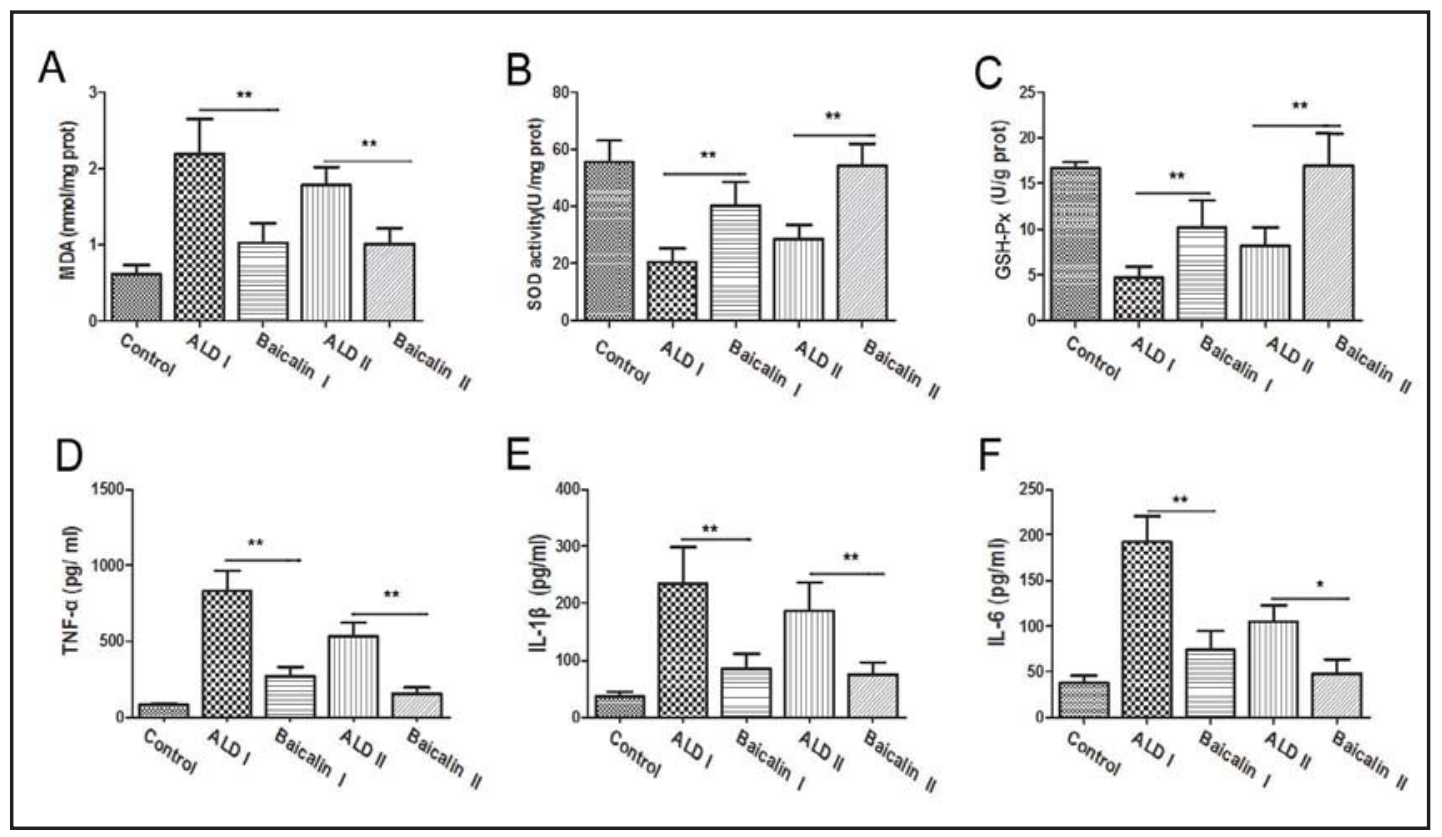

Fig. 2. Baicalin attenuates alcohol-induced oxidative stress and inflammatory cytokines production in liver. Effects of baicalin on hepatic MDA (A), SOD (B) and GSH-Px (C) expression levels in liver were measured. Effects of baicalin on TNF-a (D), IL-1 $(\mathrm{E})$, and IL-6 (F) production were determined. Data are presented as mean \pm SD. ${ }^{*} p<0.05$ and ${ }^{* *} p<0.01$ vs. ALD model group.

showed abnormal lobular architecture, evidenced by loss of hepatic lobule structure, partial loss of membrane, karyopyknosis, and fatty degeneration. In baicalin treatment groups, the hepatic structure was partially repaired. Furthermore, we found that baicalin promoted liver functional repair. The levels of serum AST, ALT, TG, and hepatic TG were significantly increased in the ALD model groups compared to those of the control group. Baicalin treatment notably reversed the elevation of these parameters (Fig. 1B-E). Biochemical results were in accord with the histology changes.

Baicalin attenuates alcohol-induced oxidative stress in liver

Long-term alcohol abuse leads to metabolism dysbolism [15]. Oxidative stress plays a critical role in the pathology of ALD [16]. To assess the effects of baicalin on alcohol-induced oxidative stress, we examined the content of MDA (a marker of lipid peroxidation) and the activities of GSH-Px and SOD in liver tissues. As shown in Fig. 2A, hepatic level of MDA was markedly increased in the ALD model groups. Synchronously, the activities of antioxidant enzymes SOD and GSH-Px were decreased compared with the control group (Fig. 2BC). Administration of baicalin increased the levels of SOD and GSH-Px and reduced MDA production in liver tissues. Together, these results indicate that baicalin alleviates alcoholinduced oxidative stress in the liver.

Baicalin inhibits pro-inflammatory cytokines production and hepatocyte apoptosis in induced by alcohol

Alcohol abuse has been reported to induce hepatic inflammation [17]. We further determined the expression of inflammatory cytokines TNF- $\alpha$, IL-1 $\beta$, and IL- 6 in liver tissues. As shown in Fig. 2D-F, significant induction of pro-inflammatory TNF- $\alpha$, IL-1 $\beta$, and IL- 6 was observed in ALD model rats. Baicalin markedly reduced TNF- $\alpha$, IL-1 $\beta$, and IL- 6 production. Furthermore, baicalin diminished the hepatotoxicity induced by alcohol. TUNEL staining showed that more TUNEL-positive cells were found in the ALD model groups compared to 


\section{Cellular Physiology Cell Physiol Biochem 2016;39:1129-1140 \begin{tabular}{ll|l} 
DOI: 10.1159/000447820 & ( ) 2016 The Author(s). Published by S. Karger AG, Basel
\end{tabular} and Biochemistry Published online: August 29, 2016 www.karger.com/cpb}

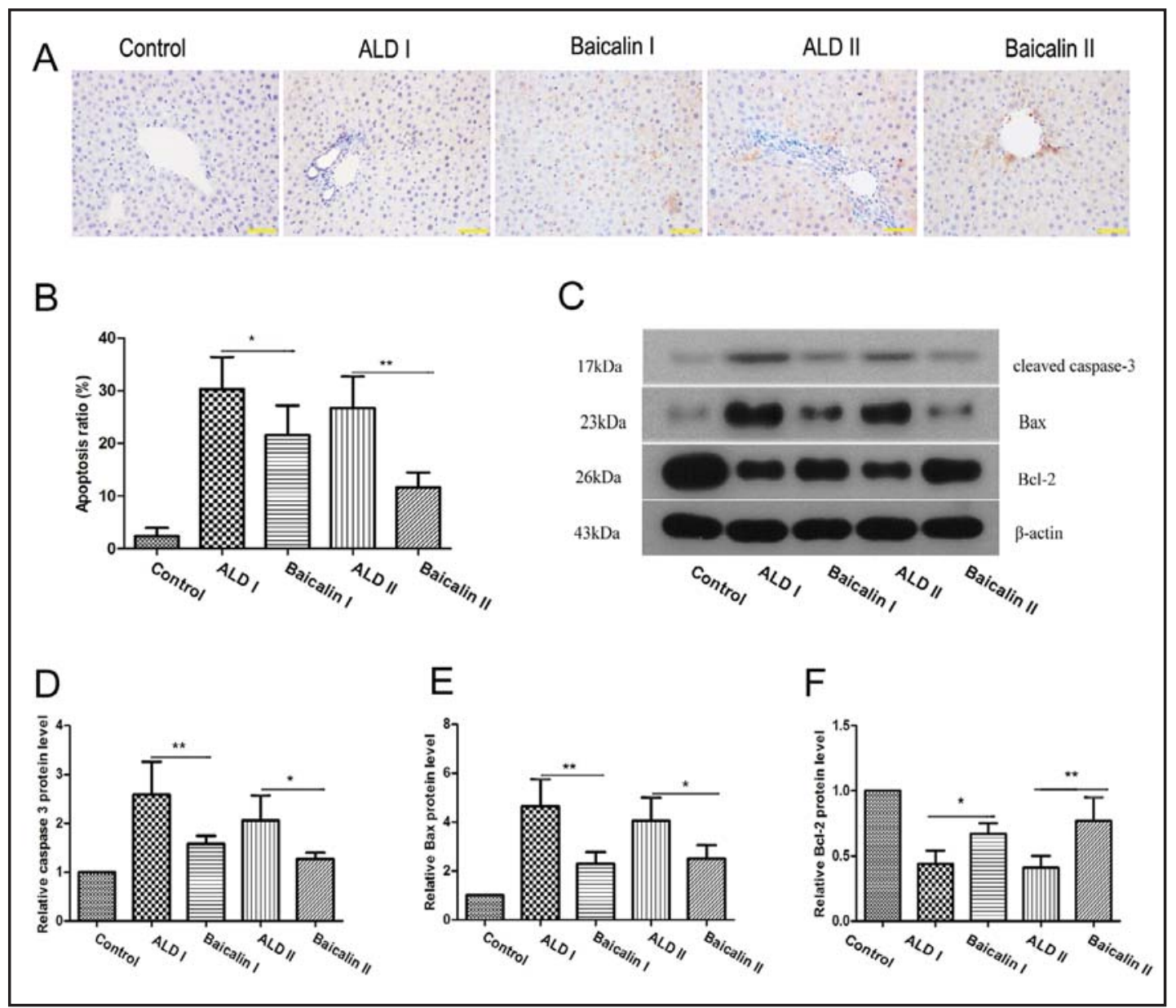

Fig. 3. Administration of baicalin inhibits hepatocyte apoptosis in alcohol-induced rats. (A-B)The apoptotic cells in the liver were assayed by TUNEL staining. (C-F) Expression levels of cleaved caspase-3, Bax, and Bcl2 were determined by Western blot analysis. $\beta$-actin was used as an internal control for grayscale analysis. Data are presented as mean \pm SD. ${ }^{*} p<0.05$ and ${ }^{* *} p<0.01$ vs. ALD model group.

the control group. However, number of apoptotic cells was reduced in the baicalin treatment groups (Fig. 3A-B). Western blot results also showed that baicalin treatment downregulated the expression of pro-apoptotic Bax and cleaved caspase-3 but increased the level of antiapoptotic Bcl-2 (Fig. 3D-F). These results indicate that baicalin inhibits inflammatory response and hepatocellular apoptosis in alcoholic liver injury.

\section{Baicalin regulates activation of the Sonic hedgehog (Shh) pathway in liver}

Sonic hedgehog (Shh) pathway plays vital roles in tissue morphogenesis and liver repair [18-20]. To assess whether baicalin affected Shh pathway in alcohol-induced liver injury, we further determined the expression of Shh pathway components including Shh ligand, patched (Ptc) and Smoothened (Smo) receptors, and Glioblastoma-(Gli-)1 transcription factor. RT-PCR and Western blot results showed that the expression of Shh, Ptc, Smo, and Gli1 were markedly increased at both mRNA and protein levels after alcoholic injury. Baicalin further promoted upregulation of these molecules (Fig. 4). Immunofluorescence assay also confirmed the increase in Shh, Ptc, Smo, and Gli-1 in the baicalin treatment groups compared to the ALD model groups (Fig. 5). Together, these results indicate that Shh signaling pathway was activated in alcohol-induced liver injury, baicalin treatment further promotes activation of the Shh pathway. 


\section{Cellular Physiology Cell Physiol Biochem 2016;39:1129-1140 \begin{tabular}{l|l|l} 
DOI: 10.1159/000447820 & (c) 2016 The Author(s). Published by S. Karger AG, Basel
\end{tabular}

A

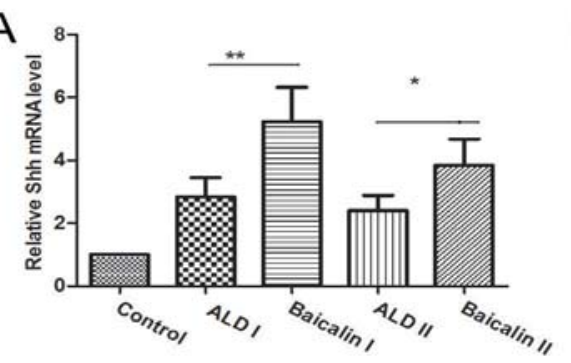

C

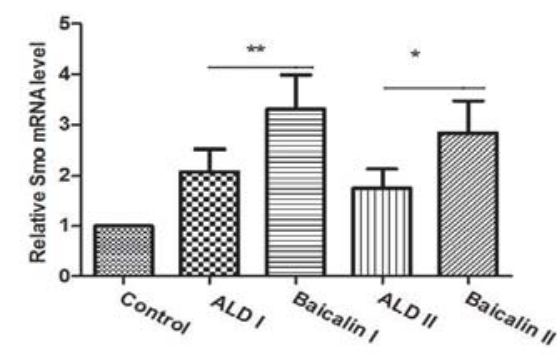

$E$

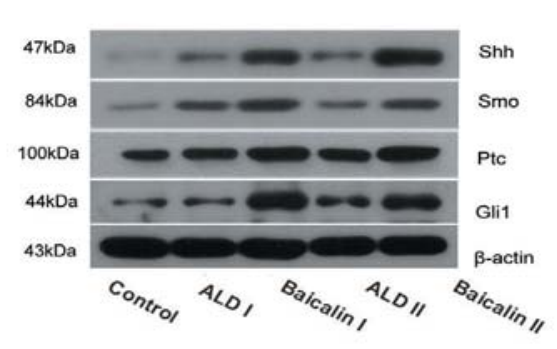

B

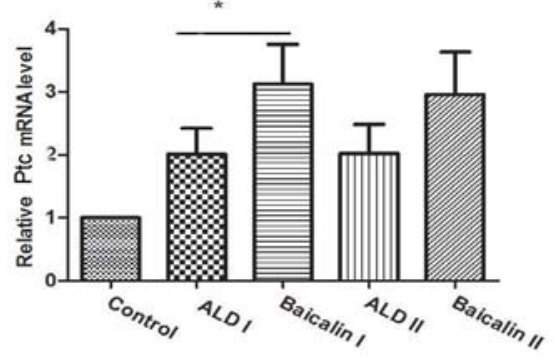

D

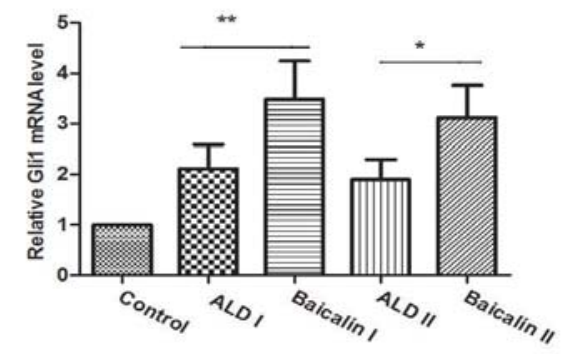

$\mathrm{F}$

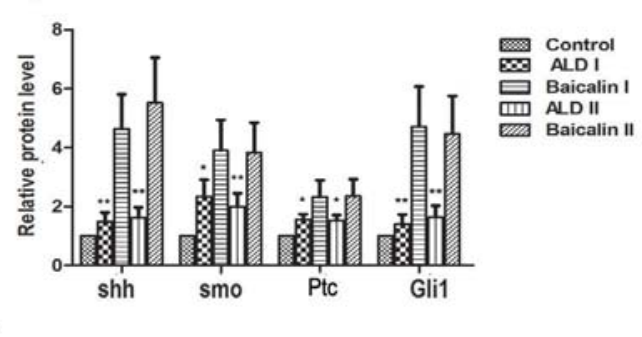

Fig. 4. Baicalin regulates the Shh signaling pathway in liver. mRNA expression of Shh (A),Ptc (B), Smo (C), and Gli1(D) in the liver were determined by RT-PCR analysis. Protein levels of Shh, Ptc, Smo, and Gli1(E-F) were determined by Western blot analysis. $\beta$-actin was used as an internal control for grayscale analysis. Data are presented as mean \pm SD. ${ }^{*} p<0.05$ and ${ }^{* *} p<0.01$ vs. ALD model group.

Baicalin mitigates alcohol-induced cytotoxicity in hepatocytes through Shh signaling pathway

To further elucidate the modulatory effect of baicalin on Shh pathway, we carried out in vitro experiments by using the Shh antagonist cyclopamine and alcohol-stimulated LO2 cells. Fig. 6A-C showed that alcohol induced high production of AST, ALT, and TG in hepatocytes, which were dose-dependently reversed by the administration of baicalin (Fig. 6A-C). Furthermore, hoechst assay revealed that DNA fragmentation with brilliant blue staining were markedly increased in alcohol-induced LO2, baicalin reduced the number of apoptotic cells (Fig. 6D). We further detected the levels of Bcl-2 and Bax. Baicalin dose-dependently increased the expression of Bcl-2, and decreased the expression of Bax and cleaved caspase 3 (Fig. 6E-F). However, the effects of baicalin were abrogated when the combination of baicalin and cyclopamine. The alcohol-induced lesion and apoptosis were aggravated after blockade of the Shh pathway when compared with the baicalin treatment group. These results suggest that the protective role of baicalin in alcohol-induced injury might be related to the Shh signaling pathway. 


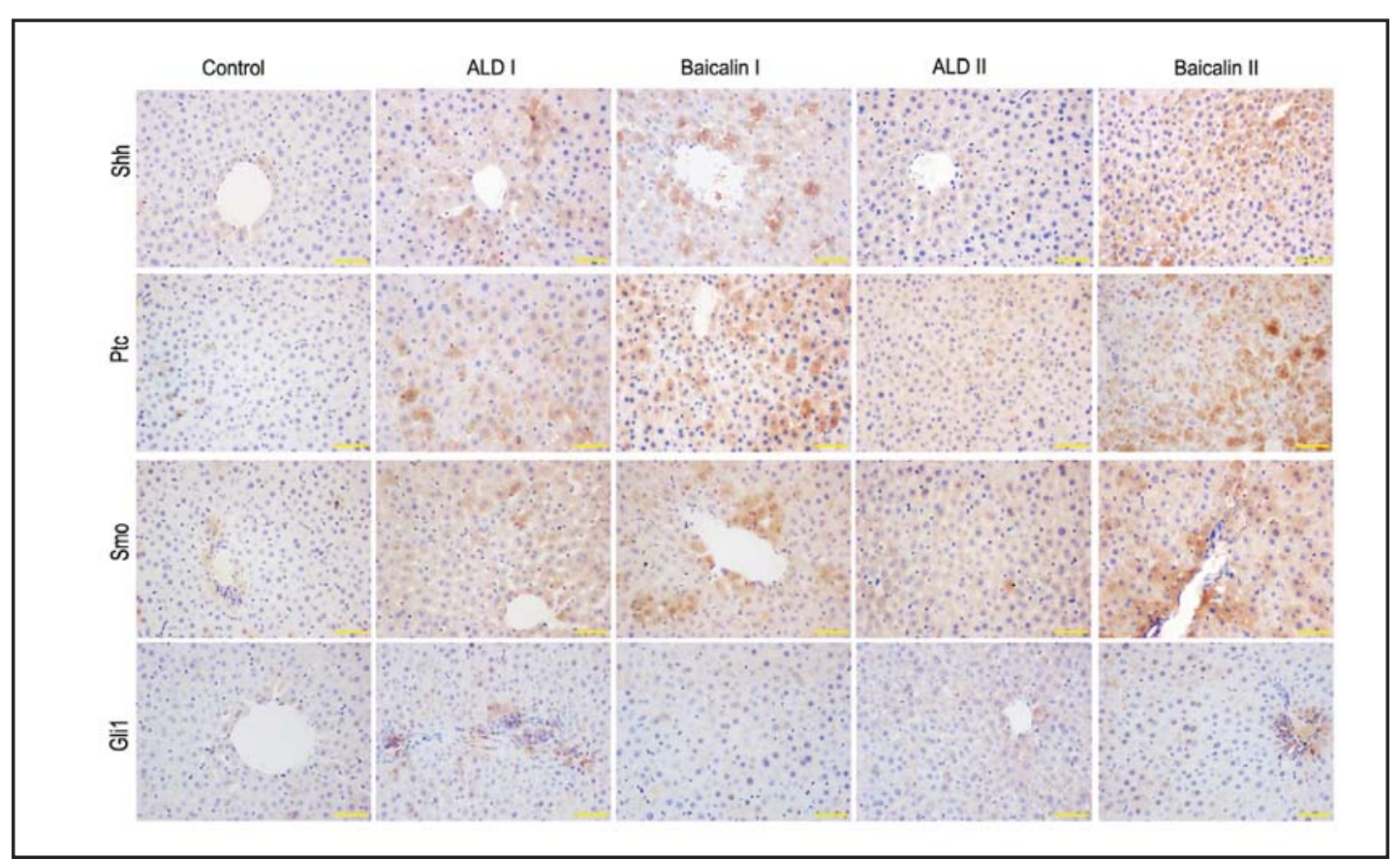

Fig. 5. Immunohistochemistry used to detect the expression level of Shh, Ptc, Smo, and Gli1 in liver from five groups. Baicalin enhances the immunoreactivity of Shh, Ptc, Smo, and Gli-1 in liver. Magnification ×400, scale bars $=50 \mu \mathrm{m}$.

\section{Discussion}

Alcohol intake is one of the most common causes of liver disease, leading to steatosis, cirrhosis, and even liver cancer. Although the mechanisms remain inconclusive, several molecular events like oxidative stress and inflammatory response have been implicated in the pathology of ALD. Thus, inhibition of these features might offer a promising therapeutic strategy for ALD. Here we demonstrate that baicalin, a polyphenolic compound isolated from Scutellaria baicalensis Georgi, ameliorates alcoholic liver injury in rats. Furthermore, we also show the direct evidence that the hepatoprotection of baicalin is probably due to the inhibition of oxidative stress and inflammatory mediators, and these effects of baicalin may attribute to the Shh signaling pathway.

Baicalin possesses a number of pharmacological activities such as anti-inflammatory, anti-oxidant, and anti-tumor properties, the therapeutic potential of baicalin has been extensively studied [21-23]. Previous studies also demonstrated that baicalin exerted hepatoprotective effect against several types of liver diseases, such as carbon tetrachlorideinduced acute hepatic injury [7], cadmium induced injury [8], and ischemia/repefusion induced liver injury[9]. To investigate whether baicalin has therapeutic efficacy in alcoholic liver injury, we first established the liver injured animal model via alcohol feeding. After continuous 4 weeks of ingestion, rats received intragastric administration of baicalin for an additional 4 weeks in the presence or absence of alcohol intake. Liver function assay showed that baicalin significantly improved the impaired liver function, as indicated by the decrease in serum ALT, AST, TG, and hepatic TG after baicalin treatment. Furthermore, baicalin alleviated pathological changes of liver caused by alcohol, suggesting a hepatoprotective action of baicalin in alcohol-induced rats.

A growing body of literature has shown that oxidative stress plays a critical role in the pathogenesis of ALD [24]. Alcohol consumption has been reported to induce free radical intermediates production like 1-hydroxyethyl radicals and ROS, and decrease the content of antioxidants. The imbalance leads to oxidative stress and tissue damage [24, 25]. Lipid peroxidation is a pathological event initiated by toxic oxygen radicals. MDA, the 


\section{Cellular Physiology \\ Cell Physiol Biochem 2016;39:1129-1140 \\ \begin{tabular}{|l|l|l}
\hline DOI: 10.1159/000447820 & (c) 2016 The Author(s). Published by S. Karger AG, Basel
\end{tabular} and Biochemistry Published online: August 29, 2016 www.karger.com/cpb

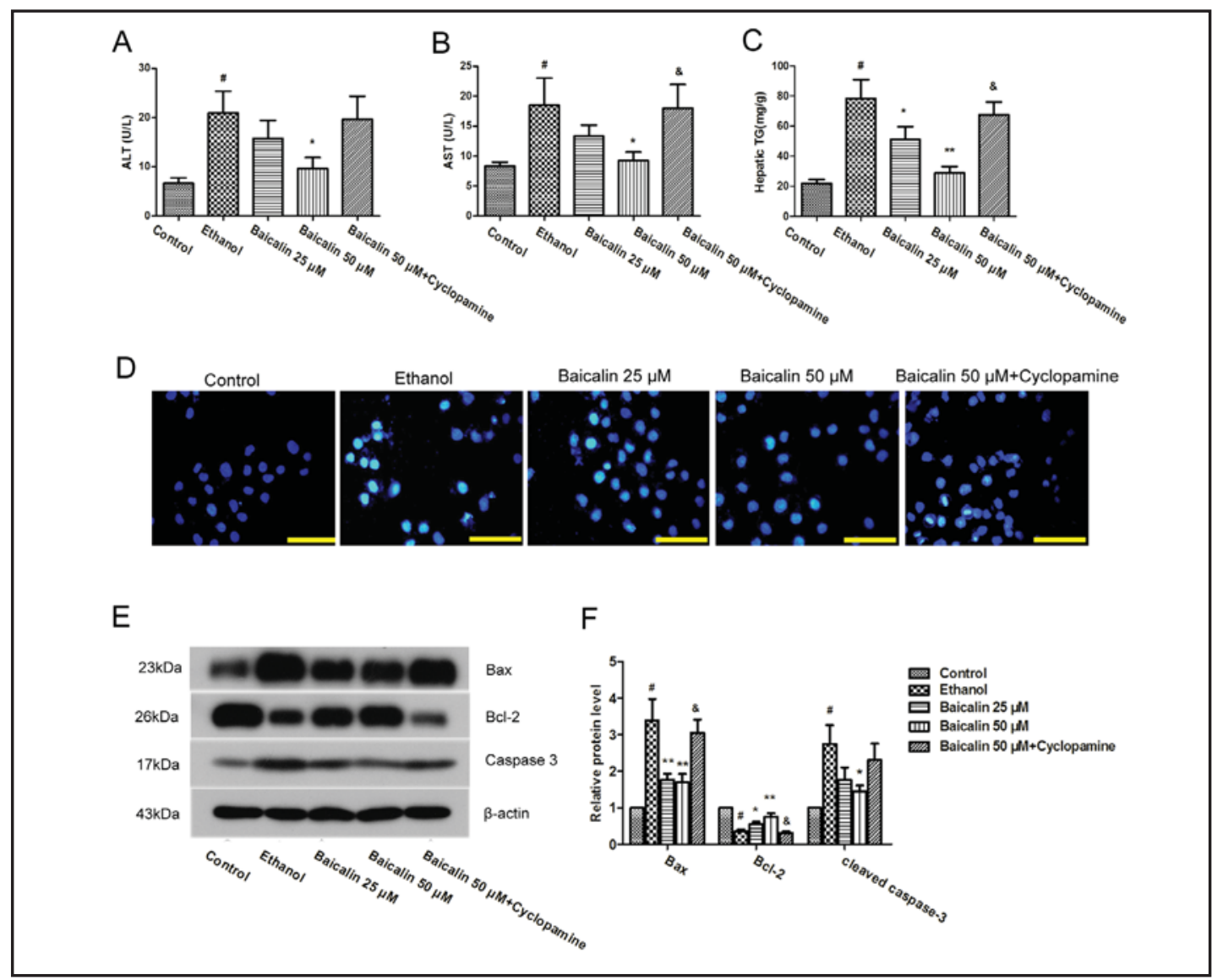

Fig. 6. Baicalin alleviated alcohol -induced injury in hepatocytes. LO2 cells were exposed to alcohol (100mM) in the absence or presence of baicalin ( $25 \mu \mathrm{M}, 50 \mu \mathrm{M}$, or $50 \mu \mathrm{M}$ plus cyclopamine) for $24 \mathrm{~h}$. The levels of (A) ALT, (B) AST in culture medium, and (C) TG in hepatocytes were determined by commercial kits. (D) Apoptotic cells were detected by Hoechst 33258 fluorescence staining. A bright blue fluorescence was shown in the nucleus of apoptotic L02. Magnification $\times 400$, scale bars $=50 \mu \mathrm{m} .{ }^{*} p<0.05$ and ${ }^{* *} p<0.01$ vs. alcohol group; \& represents $p<0.01$ vs. baicalin $50 \mu \mathrm{M}$ group; \# represents $p<0.01$ vs. control group.

main metabolite of lipid peroxidation, has been used as an indicator of ROS-induced lipid peroxidation [26]. The antioxidant effect of baicalin has been largely studied, an earlier study also reported that baicalin significantly inhibited oxidative stress in iron overload-induced liver injury [27]. Wen et al. also demonstrated that baicalin exerted antioxidatant property in cadmium induced hepatic cytotoxicity [8]. Here we detected the effect of baicalin on alcoholinduced oxidative stress by measuring MDA, GSH-Px, and SOD. Our results showed that alcohol induced significant increase in MDA expression and a decrease in antioxidant SOD and GSH-Px. Baicalin treatment effectively reversed these changes. These results indicate that the hepatoprotective function of baicalin is likely attribute to its antioxidant activity.

Inflammation is another crucial event associated with ALD progression. Alcohol abuse increases gut permeability and leading to endotoxin translocation into the circulation, which induces the production of proinflammatory cytokines such as TNF- $\alpha[28,29]$. These inflammatory mediators further result in hepatocelluar apoptosis or necrosis[30]. In the present study, we found that alcohol intake results in hepatic inflammation, as implicated by inflammatory cells infiltration and increase in pro-inflammatory cytokine TNF- $\alpha$, IL$1 \beta$, and IL- 6 expression in liver tissues. Administration of baicalin attenuated the alcoholic inflammation. Moreover, baicalin inhibited hepatocyte apoptosis and enhanced the expression of anti-apoptotic Bcl-2. Our results here were in agreement with the previous findings [22, 31]. 


\section{Cellular Physiology Cell Physiol Biochem 2016;39:1129-1140 \begin{tabular}{ll|l} 
DOI: 10.1159/000447820 & $\begin{array}{l}\text { O 2016 The Author(s). Published by S. Karger AG, Basel } \\
\text { www.karger.com/cpb }\end{array}$ \\
\hline
\end{tabular} \\ Wang et al.: Baicalin Attenuates Alcoholic Liver Injury in Rats}

The Sonic Hedgehog (Shh) signaling is an important morphogenic signaling regulating embryonic development and tissue differentiation [19, 32]. Components of Shh pathway consist of Shh ligand, Ptc and Smo receptors, and Gli transcription factors [33]. When Hh ligand interacts with Ptc receptor, inhibition of Ptch to Smo protein is removed, which can transmit downstream signal and trigger the activation of Gli family transcription factors [34]. Recently, increasing evidence demonstrates the importance of the Shh pathway in liver injury and tissue repair $[20,35]$. Activation of Hh signaling promotes the proliferation and differentiation of hepatic progenitor population [36, 37]. Furthermore, the Shh signal has been shown to affect inflammatory cytokines and oxidative stress related indexes [38, 39]. Zhou et al. reported that the Shh signal attenuated inflammation in acute pancreatitis via upregulation of IL-10 [40]. Huang et al. showed the anti-oxidative and anti-apoptotic role of Shh signal in focal cerebral ischemia model [41]. Consistent with these findings, here we found that alcohol intake activated the Shh pathway, as observed by increased expression of Shh, Smo and Ptc receptor, and transcription factor Gli1 in the liver. Baicalin treatment further enhanced the elevation of Shh components. To illuminate the modulatory action of baicalin on Shh pathway in alcoholic liver injury, we performed in vitro studies, alcohol-stimulated LO2 cells were used as an in vitro model. As expected, baicalin mitigated ehtanol-induced lesion and apoptosis of hepatocytes. However, these effects of baicalin were abolished by cyclopamine, a specific inhibitor of Shh signaling through direct interaction with SMO. Together, both in vivo and in vitro experimental results indicate that baicalin may help regeneration of hepatocytes and liver remodeling after alcoholic liver injury via Shh pathway. However, how baicalin regulates Shh signaling and is the effect of baicalin on Shh signaling involved in other molecules or signals remains to be illustrated.

In summary, our results indicate that baicalin can alleviate alcohol-induced hepatic injury in rats, the hepatoprotective action of baicalin may be due to its anti-oxidant, antiinflammatory properties, and shh signaling activation. Therefore, baicalin may be a novel therapeutic strategy ALD protection.

\section{Acknowledgments}

This study was supported by grants from the China-Japan Friendship Hospital Youth Science and Technology Excellence Project (No.: 2015-QNYC-B-02) and the Research Fund of the China-Japan Friendship Hospital (No.: 2014-2-MS-9).

\section{Disclosure Statement}

The author declares no conflict of interest.

\section{References}

1 Gao B, Bataller R: Alcoholic liver disease: pathogenesis and new therapeutic targets. Gastroenterology 2011;141:1572-1585.

2 Ezquer F, Bruna F, Calligaris S, Conget P, Ezquer M: Multipotent mesenchymal stromal cells: A promising strategy to manage alcoholic liver disease. World J Gastroenterol 2016;22:24-36.

3 Alcohol-attributable deaths and years of potential life lost--United States, 2001. MMWR Morb Mortal Wkly Rep 2004;53:866-870.

4 Gao Z, Huang K, Xu H: Protective effects of flavonoids in the roots of Scutellaria baicalensis Georgi against hydrogen peroxide-induced oxidative stress in HS-SY5Y cells. Pharmacol Res 2001;43:173-178.

5 Tang YJ, Zhou FW, Luo ZQ, Li XZ, Yan HM, Wang MJ, Huang FR, Yue SJ: Multiple therapeutic effects of adjunctive baicalin therapy in experimental bacterial meningitis. Inflammation 2010;33:180-188. 


\section{Cellular Physiology Cell Physiol Biochem 2016;39:1129-1140 \begin{tabular}{ll|l}
\cline { 2 - 3 } DOI: 10.1159/000447820 & ( ) 2016 The Author(s). Published by S. Karger AG, Basel
\end{tabular} and Biochemistry Published online: August 29, 2016 www.karger.com/cpb}

Wang et al.: Baicalin Attenuates Alcoholic Liver Injury in Rats

6 Liao P, Liu L, Wang B, Li W, Fang X, Guan S: Baicalin and geniposide attenuate atherosclerosis involving lipids regulation and immunoregulation in ApoE-/- mice. Eur J Pharmacol 2014;740:488-495.

7 Park SW, Lee CH, Kim YS, Kang SS, Jeon SJ, Son KH, Lee SM: Protective effect of baicalin against carbon tetrachloride-induced acute hepatic injury in mice. J Pharmacol Sci 2008;106:136-143.

8 Wen YF, Zhao JQ Bhadauria M, Nirala SK: Baicalin prevents cadmium induced hepatic cytotoxicity, oxidative stress and histomorphometric alterations. Exp Toxicol Pathol 2013;65:189-196.

9 Kim SJ, Moon YJ, Lee SM: Protective effects of baicalin against ischemia/reperfusion injury in rat liver. J Nat Prod 2010;73:2003-2008.

10 Kim SJ, Lee SM: Effect of baicalin on toll-like receptor 4-mediated ischemia/reperfusion inflammatory responses in alcoholic fatty liver condition. Toxicol Appl Pharmacol 2012;258:43-50.

11 Feng J, Guo C, Zhu Y, Pang L, Yang Z, Zou Y, Zheng X: Baicalin down regulates the expression of TLR4 and NFkB-p65 in colon tissue in mice with colitis induced by dextran sulfate sodium. Int J Clin Exp Med 2014;7:4063-4072.

12 Hochhauser E, Lahat E, Sultan M, Pappo O, Waldman M, Sarne Y, Shainberg A, Gutman M, Safran M, Ben Ari Z: Ultra Low Dose Delta 9-Tetrahydrocannabinol Protects Mouse Liver from Ischemia Reperfusion Injury. Cell Physiol Biochem 2015;36:1971-1981.

13 Abdelazim SA, Darwish HA, Ali SA, Rizk MZ, Kadry MO: Potential antifibrotic and angiostatic impact of idebenone, carnosine and vitamin $\mathrm{E}$ in nano-sized titanium dioxide-induced liver injury. Cell Physiol Biochem 2015;35:2402-2411.

14 Nyblom H, Berggren U, Balldin J, Olsson R: High AST/ALT ratio may indicate advanced alcoholic liver disease rather than heavy drinking. Alcohol Alcohol 2004;39:336-339.

15 Ambade A, Mandrekar P: Oxidative stress and inflammation: essential partners in alcoholic liver disease. Int J Hepatol 2012;2012:853175.

16 Apel K, Hirt H: Reactive oxygen species: metabolism, oxidative stress, and signal transduction. Annu Rev Plant Biol 2004;55:373-399.

17 Lu C, Xu W, Zhang F, Jin H, Chen Q, Chen L, Shao J, Wu L, Lu Y, Zheng S: Ligustrazine prevents alcoholinduced liver injury by attenuating hepatic steatosis and oxidative stress. Int Immunopharmacol 2015;29:613-621.

18 Wang S, Hyun J, Youn B, Jung Y: Hedgehog signaling regulates the repair response in mouse liver damaged by irradiation. Radiat Res 2013;179:69-75.

19 Ingham PW, McMahon AP: Hedgehog signaling in animal development: paradigms and principles. Genes Dev 2001;15:3059-3087.

20 Omenetti A, Choi S, Michelotti G, Diehl AM: Hedgehog signaling in the liver. J Hepatol 2011;54:366-373.

21 Xi Y, Wu M, Li H, Dong S, Luo E, Gu M, Shen X, Jiang Y, Liu Y, Liu H: Baicalin Attenuates High Fat DietInduced Obesity and Liver Dysfunction: Dose-Response and Potential Role of CaMKKbeta/AMPK/ACC Pathway. Cell Physiol Biochem 2015;35:2349-2359.

22 Cui L, Feng L, Zhang ZH, Jia XB: The anti-inflammation effect of baicalin on experimental colitis through inhibiting TLR4/NF-kappaB pathway activation. Int Immunopharmacol 2014;23:294-303.

23 Wang H, Liu D: Baicalin inhibits high-mobility group box 1 release and improves survival in experimental sepsis. Shock 2014;41:324-330.

24 Albano E: Alcohol, oxidative stress and free radical damage. Proc Nutr Soc 2006;65:278-290.

25 Scott RB, Reddy KS, Husain K, Schlorff EC, Rybak LP, Somani SM: Dose response of ethanol on antioxidant defense system of liver, lung, and kidney in rat. Pathophysiology 2000;7:25-32.

26 Singh M, Kapoor A, Bhatnagar A: Oxidative and reductive metabolism of lipid-peroxidation derived carbonyls. Chem Biol Interact 2015;234:261-273.

27 Zhang Y, Huang Y, Deng X, Xu Y, Gao Z, Li H: Iron overload-induced rat liver injury: Involvement of protein tyrosine nitration and the effect of baicalin. Eur J Pharmacol 2012;680:95-101.

28 Mir H, Meena AS, Chaudhry KK, Shukla PK, Gangwar R, Manda B, Padala MK, Shen L, Turner JR, Dietrich P, Dragatsis I, Rao R: Occludin deficiency promotes ethanol-induced disruption of colonic epithelial junctions, gut barrier dysfunction and liver damage in mice. Biochim Biophys Acta 2016;1860:765-774.

29 Rao RK, Seth A, Sheth P: Recent Advances in Alcoholic Liver Disease I. Role of intestinal permeability and endotoxemia in alcoholic liver disease. Am J Physiol Gastrointest Liver Physiol 2004;286:G881-884. 


\section{Cellular Physiology Cell Physiol Biochem 2016;39:1129-1140 \begin{tabular}{ll|l} 
DOI: 10.1159/000447820 & O 2016 The Author(s). Published by S. Karger AG, Basel \\
and Biochemistryarger.com/cpb
\end{tabular}}

Wang et al.: Baicalin Attenuates Alcoholic Liver Injury in Rats

30 Luo M, Zhao A, Li J, Chen Y, Tian D, Wang C, Hu Z, Gao J: Acute liver injury attenuation of a novel recombinant sTNFR through blocking hepatic apoptosis. Immunopharmacol Immunotoxicol 2015;37:295300.

31 Zhu J, Wang J, Sheng Y, Zou Y, Bo L, Wang F, Lou J, Fan X, Bao R, Wu Y, Chen F, Deng X, Li J: Baicalin improves survival in a murine model of polymicrobial sepsis via suppressing inflammatory response and lymphocyte apoptosis. PLoS One 2012; 7:e35523.

32 Lin SL, Chang SJ, Ying SY: Transcriptional control of Shh/Ptc1 signaling in embryonic development. Gene 2006;367:56-65.

33 Varjosalo M, Li SP, Taipale J: Divergence of hedgehog signal transduction mechanism between Drosophila and mammals. Dev Cell 2006;10:177-186.

34 Briscoe J, Therond PP: The mechanisms of Hedgehog signalling and its roles in development and disease. Nat Rev Mol Cell Biol 2013;14:416-429.

35 Cai Y, Zheng H, Gong W, Che Y, Jiang B: The role of hedgehog signaling pathway in liver regeneration. Hepatogastroenterology 2011;58:2071-2076.

36 Deutsch G, Jung J, Zheng M, Lora J, Zaret KS: A bipotential precursor population for pancreas and liver within the embryonic endoderm. Development 2001;128:871-881.

37 Omenetti A, Diehl AM: The adventures of sonic hedgehog in development and repair. II. Sonic hedgehog and liver development, inflammation, and cancer. Am J Physiol Gastrointest Liver Physiol 2008;294:G595598.

38 Dai RL, Zhu SY, Xia YP, Mao L, Mei YW, Yao YF, Xue YM, Hu B: Sonic hedgehog protects cortical neurons against oxidative stress. Neurochem Res 2011;36:67-75.

39 Marwaha S, Schumacher MA, Zavros Y, Eghbalnia HR: Crosstalks between cytokines and Sonic Hedgehog in Helicobacter pylori infection: a mathematical model. PLoS One 2014;9:e111338.

40 Zhou X, Liu Z, Jang F, Xiang C, Li Y, He Y: Autocrine Sonic hedgehog attenuates inflammation in ceruleininduced acute pancreatitis in mice via upregulation of IL-10. PLoS One 2012;7:e44121.

41 Huang SS, Cheng H, Tang CM, Nien MW, Huang YS, Lee IH, Yin JH, Kuo TB, Yang CC, Tsai SK, Yang DI: Antioxidative, anti-apoptotic, and pro-angiogenic effects mediate functional improvement by sonic hedgehog against focal cerebral ischemia in rats. Exp Neurol 2013;247:680-688. 